\title{
Medicinal herbs in the management of male infertility
}

\author{
Sulagna Dutta and Pallav Sengupta* \\ Department of Physiology, MAHSA University, Malaysia
}

\begin{abstract}
Male infertility can range from neurohormonal imbalances, reproductive tissue disruptions, qualitative and quantitative deterioration of semen to sexual behavioral problems. The global deterioration of male reproductive health is a major concern and the modern therapeutic approaches to combat male infertility are expensive, less accessible, have long term treatment tenure and possess various side effects. Whereas, the herbal therapies, are better positioned to offer more holistic approaches to improve male reproductive health. In Ayurvedic pharmacology, there exists a special group of herbs, classified as vajikarana or aphrodisiacs, which nourishes and stimulates the sexual tissues. This review concentrates on the Ayurvedic approach to ameliorate male reproductive health referring to some of the most important scientifically tested herbs which have been reported to accentuate male fertility by imposing either stimulating or nourishing effects on the male reproductive organs.
\end{abstract}

\section{Introduction}

Organisms strive to exist and preserve own genetic characteristics through subsequent progenies owing to their reproductive potencies. In this aspect, human being is one of the most successful survivors on earth. But the present scenario is alarming, as besides female reproductive complications and dysfunctions, male reproductive health is suffering a deteriorating trend throughout the globe [16]. Sexual dysfunction, an earnest medical issue marring social and biological relationships, occurs in $25 \%-63 \%$ of women and $10 \%-52 \%$ in men. Sexual dysfunction in men can be explained as the failure to accomplish normal sexual intercourse, inability to respond to erectile stimuli or sustain an erection, retarded ejaculation, decreased libido, unregulated sexual behavior etc. [7]. Innumerable synthetic products are abundantly floating in the global market with quick remedial claims. But most of them are associated with suppressing symptoms and side effects [8]. Thereby people are again oriented back to herbal and ayurvedic aphrodisiacs having the holistic approach to cure male reproductive problems from the root of the causes.

Aphrodisiacs, agents to arouse sexual desire and performance, when derived from herbs, have been observed to directly been able to excite male sexual libido, contribute to sustenance of reproductive activities, restore healthy tissue functioning and also aid the neuroendocrine regulation for exhibiting required sexual strength with content state of mind and body [9]. This review, with the aim to highlight the healthy longterm remedy of male sexual problems, concentrates on the scientifically tested herbs which have been reported to accentuate male fertility.

\section{Overview of male reproductive disruptions}

Standard clinical and laboratory evaluations are not enough to find out the causes behind most of the male infertility cases [10]. It can range from hormonal or neuronal imbalances, reproductive tissue disruptions to qualitative and quantitative deterioration of semen. Oxidative stress induced by reactive oxygen species (ROS) is a major causative factor in male infertility $[11,12]$. The mature spermatozoa are encased in polyunsaturated lipid membrane which is vulnerable to the oxidative damage induced by ROS, and this, in turn, can impair spermatogenesis and reduce sperm quality, motility and morphology $[10,14]$. The regulation of hypothalamic-pituitary-gonadal (HPG) axis is the main controller of male reproductive function where the hypothalamus produces gonadotropin-releasing hormone $(\mathrm{GnRH})$ to stimulate the secretion of follicle-stimulating hormone (FSH) and luteinizing hormone (LH) from the anterior pituitary. The secreted FSH and LH act on the Sertoli cells and Leydig cells, respectively to stimulate spermatogenesis and testosterone [11,14,15]. Spermatogenesis is regulated by well-maintained crosstalk of $\mathrm{FSH}, \mathrm{LH}$, intratesticular testosterone and other hormones. Thereby, HPG axis disruptions are detrimental to semen parameters $[16,17]$.

Reproductive endocrinologist opts for injectable medications including recombinant $\mathrm{FSH}$, human menopausal gonadotropin (hMG), human chorionic gonadotropin (hCG) and gonadotropin-releasing hormone $(\mathrm{GnRH})$ to treat men with abnormal semen parameters. An oral medication, Clomiphene citrate which is an estrogen receptor antagonist, is also given to the patient in order stimulate gonadotropin release from the pituitary. But these options are expensive, less accessible, having long term treatment tenure and possess various sideeffects [18]. Whereas, the herbal therapies, are better positioned to offer more holistic approaches to improve male reproductive health.

Correspondence to: Pallav Sengupta, Ph.D., Senior Lecturer, Department of Physiology, Faculty of Medicine, MAHSA University, Jalan SP 2, Jalan Saujana Putra, 42610, Jenjarum, Selangor, Malaysia, Phone: +60 174523780 E-mail: pallav_cu@yahoo.com

Key words: aphrodisiac, erectile dysfunction, male infertility, testosterone

Received: February 03, 2018; Accepted: February 25, 2018; Published: February 28,2018 


\section{Herbal therapy and male reproductive health}

According to the Ayurvedic concept, health depends upon balance between three fundamental bodily bio-elements or doshas referred to as Vata, Pitta and Kapha [19]

Vāta or Vata (airy element), characterised by dry, cold, light, minute, and movement.

Pitta (fiery element) or bile, flowing through the liver and permeating the overall heating of the body.

Kapha (watery element) is characterised by heaviness, cold, tenderness, slowness, lubrication, nutrient carrier, nourishment.

In Ayurvedic pharmacology, there exists a special group belonging to rasayana herbs, classified as vajikarana, that aid nourishment and also stimulate the sexual tissues. Moreover, they also help to promote beauty and sex appeal. Further classifications of herbs that increase spermatogenesis, are known as shukrala. With the knowledge of the possible causes and factors leading to male infertility, the appropriate herb can be selected to meet the need [19].

In Sanskrit 'vaji' and 'karana' mean 'horse' and 'power' respectively, in order to convey the notion about the power or strength that a horse possesses. These are the herbs which can be referred to in Western term as aphrodisiacs. Vajikarana herbs can be used as stimulants or tonics to improve male reproductive vitality. Stimulants to reproductive functions are typically heating substances like fenugreek, garlic, damiana, and onion. They have an invigorating action on the male sexual organs. Tonics provide better nourishment to the reproductive tissues to rejuvenate the quality and quantity of the same. Some herbs have both stimulating as well as nourishing properties, such as garlic, fenugreek, shilajit, and ashwagandha (Withania somnifera).

The Ayurvedic herbal drugs can be classified in varied ways, and one such simplified classification according to their functions on male reproductive health is as follows [20]:

Herbs increasing the quantity of semen or stimulating semen production, for example, Polygonatum verticillatum, Mucuna pruriens, Microstylis wallichii, Roscoea procera, and Asparagus racemosus.

Herbs purifying and improving semen quality, for example, Vetiveria zizanioides, Saussurea lappa, Myrica nagi, Sesamum indicum, and Anthocephalus cadamba.

Herbs rejuvenating ejaculatory functions, for example, Strychnos nux vomica, Myristica fragrans, Cannabis sativa, and Cassia occidentalis.

Herbs improving sustenance and ejaculatory performance, for example, Cinnamomum tamala, Anacyclus pyrethrum, Sida cordifolia, Asparagus racemosus, Mucuna pruriens, and Cannabis sativum.

Herbs increasing libido, namely, Asparagus racemosus, Withania somnifera, Datura stramonium, Anacyclus pyrethrum, Hibiscus abelmoschus, and Opium.

\section{Some common Ayurvedic herbs to improve male fertility}

\section{Ashwagandha (Withania somnifera)}

In Sanskrit, ashwagandha refers to 'the smell of a horse', owing to its root's strong odor resembling that of horse urine. Another reason for its naming for mythological comparison of the sexual vitality it may impart to men with that of a horse. Innumerable beneficial qualities to alleviate male reproductive disorders including induction of spermatogenesis, improved blood flow to the reproductive tissues and regulated endocrine functions. It relieves conditions such as spermatorrhea, nocturnal emission, premature ejaculation, and enlarged prostate.

Withania somnifera is able to combat stress-induced male infertility and protects against swimming-induced endocrine dysfunctions of male reproduction in rat [21]. The aqueous extract of this herb has been shown to improve spermatogenesis, owing to elevated interstitial cell stimulating hormone and testosterone-mimicking effects together with induction of nitric oxide synthase [22]. Yet another study showed that Ashwagandha root extract administration resulted in a significantly greater improvement in spermatogenic activity and increase in serum hormone levels in the oligospermic patients as compared to the placebo treated [23]. This herb evidently combats against oxidative stress by reducing lipid peroxidation as well as protein carbonyl content, increasing sperm count and motility, rejuvenating the seminal plasma levels of antioxidant enzymes. Withania somnifera root powder when administered to normozoospermic infertile man in a dosage of $5 \mathrm{~g} /$ day for 3 months showed a significant reduction in stress, improved antioxidants, and enhanced semen volume and levels of vitamins A, $\mathrm{C}$, and $\mathrm{E}$ and also corrected fructose. This herb's action at the HPG axis level is also evident through various studies, one such showed significant increased serum testosterone and LH levels and decreased levels of FSH and prolactin (PRL) in infertile men [24-26].

In terms of ayurvedic explanations, the grounding and deeply nourishing qualities of Withania somnifera contribute for its being one of the best vata appeasing medicines. Its warming energy provokes pitta if used in excess and so it is recommended to combine it with cooling vajikarana herbs such as licorice, vidari, shatavari, bala, while using as a drug. Owing to it stress relieving, relaxing effect on the body and mind, as well as fortifying of all the dhatus, it is considered as the best herb to target many of the contributing factors that are associated with reduced sexual energy.

\section{Kapikacchu (Mucuna pruriens)}

This sweet yet sometimes bitter tasted herb is quite heavy and oily. Mucuna pruriens is mainly used to balance vata and pitta, as both $k a p h a$ and $a m a$ are increased in excess by this herb. Typical doses of the powdered Mucuna pruriens range from 1.5 to 6 grams [27]. Alkaloids derived from $M$. pruriens seeds were found to stimulate spermatogenesis and overall weight of the testicles and accessory glands in the male albino rat [28]. M. pruriens is capable of stimulating sexual activities in male rats as inferred by increased mounting frequency, ejaculation latency and also enhanced intromission frequency [29,30]. In a study, $M$. pruriens has been shown to efficiently recover the spermatogenic loss which was induced in male rats by ethinyl estradiol administration and the herb's beneficial effects were mediated by mitigation of ROS, apoptosis regulation, and increase in the number of germ cells. The major constituent of $M$. pruriens, $L-D O P A$, largely contributes to its pro-spermatogenic properties [31]. The seed extract of $M$. pruriens showed marked improvement in sexual potency and behavior, libido, sperm parameters, and endocrine levels [32]. M. pruriens has been able to ameliorate the levels lipids, triglycerides, cholesterol, phospholipids, vitamin $\mathrm{A}, \mathrm{C}$, and $\mathrm{E}$ and corrected fructose nullifying oxidative stress induced lipid peroxidation in seminal vesicles and restored the levels of SOD, catalase, GSH, and ascorbic acid in seminal plasma [33, 34]. From the neuroendocrine perspective, $M$. pruriens elevates testosterone, $\mathrm{LH}$, dopamine, adrenaline, as well as noradrenaline levels and decreased 
levels of FSH and PRL in infertile men. M. pruriens treatment to infertile men indeed improves steroidogenesis and semen quality [35].

\section{Shatavari (Asparagus racemosus)}

Shatavari can serve as a powerful male tonic. With a bittersweet taste, this herb renders cooling and purifying effect to the liver and blood, and targets pitta at its main site in the small intestine. Its cooling properties balance the heating herbs which are used to improve sperm count, such as, garlic, onion, ashwagandha, etc. Thus, Shatavari prevents depletion of sperm caused by burning via excess pitta. Owing to its heavy and nourishing properties, it is useful for vata, especially when combined with heating vajikaranas like ashwagandha or bala. As a drug, it can be prepared as a milk decoction with 3 to 6 grams of the herb, or can be taken alone or in combination with other herbs [19]. Sexual desires are associated with emotional aspects and in such conditions, this herb in combination with cooling nervine herbs like brahmi (gotu kola) may enhance libido and help to overcome agonizing emotions like anger and irritability. Shatavari is thus one of best pitta pratyanika herbs which can be associated in several Ayurvedic formulas to balance pitta and vata in mediating male reproductive functions. General fatigue, low sexual energy, anger, stress, irritability, inflammation, hyperacidity, urogenital infections, burning sensations etc. are the conditions when this herb can be used as drug in standardized dosage of 2 to 6 grams of the powdered herb, 2 to 3 times daily. This herb is avoided in cases of respiratory or sinus congestion [19].

\section{Bala (Sida cordifolia)}

Bala refers to 'strength' in Sanskrit, and being a rejuvenative tonic herb for vata and pitta, this herb finds application in nourishment and strengthening of all the bodily tissues, especially the muscle, plasma, muscle, bone marrow and reproductive tissue. It is sweet, heavy and oily and increase kapha when used in excess, and also is mildly cooling. Bala is one of the best anti-vata herbs in Ayurveda which mitigates disorders related to both body and mind. It plays a leading role as an Ayurvedic herb in the treatment of balakshaya or chronic fatigue, to rejuvenate overall health in exhaustion of physical or mental strength. It serves as a tonic to restore sexual strength, promote spermatogenesis and enhance male fertility $[19,20]$.

This herb can be taken internally or even used as massaging oil in combination with Ashwagandha onto penis to improve its tone and also to prevent premature ejaculation. Its combination with herbs such as gokshura, ashwagandha, vidarikandha, saw palmetto, and kapikacchu, proved to be beneficial for prostate health. A typical dose of Bala ranges from 2 to 6 grams, to be taken 2 to 3 times daily [19].

\section{Vidarikandha (Ipomoea digitata)}

Vidarikandha is a starchy tuber, effective in promoting spermatogenesis with faster action when taken as a milk decoction. It is lighter for kapha types than shatavari and bala. Its combination with kapikacchu is effective in the treatment of enlarged prostate. This sweet and cool herb promotes ojas, muscle tone, motor coordination and nullifies sexual debility associated with nervousness and adrenal stress when used in formulation with ginseng, licorice, gokshura, and ashwagandha. It falls right between too warming and too cooling herbs providing great value as both a vata and pitta balancing herb. A typical dose of Vidarikandha ranges from 2 to 6 grams, 2 to 3 times daily [19].

\section{Shilajit (Asphaltum, mineral pitch)}

Shilajit is immensely important Ayurvedic herb which, with its heating energy, increases virility and sexual stamina via removing excess kapha, while maintaining the normal genital tone. In kapha related reproductive imbalances, it is used in combination with Ashwagandha. For enlarged prostate, its combination with saw palmetto, punarnava, gokshuradiguggulu, ashwagandha, or vidhari, are effective herbal remedies. This herb should be used in doses ranging from 250 to 500 mg twice daily [19].

\section{Pippali (Piper longum)}

Pippali is a rejuvenating herb with a warming, stimulating and kapha reducing action. Its oily nature prevents it from drying making it suitable for vata and its pleasant post digestive effect makes it more calming to pitta than other hot spices and herbs. It aids blood flow to the reproductive tissues when taken in combination with Ashwagandha $[19,20]$.

According to the Ayurveda, Pippali enhances life energy and expels impurities via respiration. It also improves digestion, absorption, assimilation, respiration and reduces arthritic agonies. The typical dosage of this herb is 1 to 3 parts in complex formulas, or ranges from $250 \mathrm{mg}$ to 1.5 grams. Pippali should be avoided in high pitta or inflammatory conditions [19].

\section{Butea superba}

Buteasuperba Roxb (Leguminosae) or the 'Red KwaoKrua', has long been consumed as a stimulant of male sexual vigor. Its alcoholic extract $(0.01,0.1$ or $1.0 \mathrm{mg} / \mathrm{kg}$ BW/day) for 6 months significantly elevted the sperm concentration and enhanced sperm motility with no disruptive signs to sperm or testis [36]. Powdered crude extract of this herb at the doses of 2,25,250, and $1250 \mathrm{mg} / \mathrm{kg}$ body weight in male rats for 8 weeks increased testis weight and sperm counts [37]. Moreover, the ethanol extract of $B$. superba has been shown to increase penile erection acting through cAMP/cGMP pathways [38].

\section{Curculigo orchioides}

Curculigo orchioides Gaertn (Amaryllidaceae), or Kali Musli or Syah (black) Musli, is an aphrodisiac or rejuvenator ameliorating sexual arousal and performance by inducing penile erection, mating sustenance, orientation behavior, etc. This herb also promotes anabolic and spermatogenic effect by pronounced increased weight of reproductive organs. Rats that received Curculigo orchioides treatment displayed reduction in mount latency, increment in penile erection index, and increase in mount frequency and affinity towards female [39,40].

Its lyophilized aqueous extracts are shown to improve the pendiculatory activity in male rats after as well as preserve the in vitro sperm after $30 \mathrm{~min}$. of incubation [41]. The aqueous extract of the herb is effective at a dose of $200 \mathrm{mg} / \mathrm{kg}$. In case of physically induced or heat induced sexual dysfunction, this herb is useful in ameliorating the decreased spermatogenesis and mitigated disruptions owing to the heat shock protein [42].

\section{Cynomorium coccineum}

Cynomorium coccineum Linn. (Cynomoraceae) is a black leafless parasitic plant without chlorophyll. Aqueous extract of this herb has been shown to induce significant elevation in the sperm count, the percentage of viable sperm and sperm motility, reduced the number of abnormal sperm, increased spermatogenesis [43].

\section{Chlorophytum borivilianum}

Safed Musli (Chlorophytum borivilianum) of family Liliaceae is claimed to be an effective aphrodisiac and sexual stimulant. Ethanolic 
roots extract of this herb or sapogenins extracted from the roots showed anabolic and spermatogenic effect in treated male rats evidenced by total body weight increase and also increment in weight of reproductive organs. The herb could also affect sexual behavior of animals by reducing mount ejaculation, post-ejaculatory latency, and intromission latency, increasing mount frequency and affinity towards female [44]. Moreover, the aqueous extract of dried roots of this herb enhanced sexual arousal, strength, and libido as well as sperm count in Wistar male rats [45]. This herb also reportedly improves male reproductive functions in diabetic scenario $[46,47]$.

\section{Epimedium koreanum}

Epimedium L. (Berberidaceae), is a popular Chinese herb and botanical supplement used as health tonic. Most important species of this herb used for medicinal purposes are E. pubescensMaxim., E. brevicornum Maxim, E. koreanumNakai, E. sagittatum (Sieb. EtZucc) Maxim, and E. wushanense T.S. Ying [48]. Hydroalcholic extract of the herb shown to display aphrodisiac effects and are commonly used to enhance erectile function [49]. The primary active component of Epimedium extracts, Icariin, is a type of flavonoid and selectively inhibits phosphodiesterase-5 (PDE5) improving erectile function [50]. Icariin also can mimic the effects of testosterone inducing spermatogenesis [51].

\section{Eurycoma longifolia}

Eurycoma longifolia Jack (Simaroubaceace) or Tongkat Ali, is one of the major aphrodisiac herb from Malaysia [52]. E. longifolia extract treatment showed successful induction of sexual motivation in naive male rats [53]. Ethanol extract of this herb increased the sexual libido and performance of the treated male rats by increasing the duration of coitus and copulation frequency [54].

\section{Tribulus terrestris}

Tribulus terrestris Linn. (Zygophyllaceae) or puncture vine, a perennial creeping herb, has worldwide distribution and is regarded as an aphrodisiac since ancient times besides being used for various ailments such as inflammations, leucorrhoea, urinary infections, edema, and ascites [55]. Administration of T. terrestris to animals showed to improve plasma testosterone level and induced spermatogenesis [56]. It also increases the levels of testosterone as well as luteinizing hormone [57] and the activities of dehydroepiandrosterone, dihydrotestosterone, and dehydroepiandrosteronesulphate [58]. The corpus cavernosal tissues of New Zealand white rabbits following treatment with this herb were tested in vitro with various pharmacological agents as well as electrical field stimulation and the herb was shown to have proerectile effect [59]. It increases sexual behavior evident through increase in mount frequency and intromission frequency, reduction in mount latency, intromission latency, and penile erection index as well as increase in prostate weight and intracavernosal pressure [60,61]. This herb also possesses androgen, testosterone, dihydrotestosterone (DHT), and Ddehydroepiandrosterone (DHEA) increasing property having stimulating effects on reproductive functions and thus used in mild to moderate cases of endocrine disruptions $[62,63]$. The ability of to induce nitric oxide release may also account for its claims as an aphrodisiac [64].

\section{Mechanism of action}

Male reproductive functions are restored and controlled by induction or inhibition of neurochemicals, regulation of neuroendocrine axis and their cross talks as well as by local mediators acting within the reproductive tissues [65-67]. The herbal therapies may ameliorate male reproductive functions possibly at three levels. (1) Herbs may act on the central or peripheral nervous system improving the responsiveness of male reproductive tissues. The neuronal control over the male reproductive organs is mediated by an orchestra of neurochemicals/neurotransmitters, among which the serotonin and dopamine are the prime controllers of sexual behavior with serotonin mediating inhibitory functions and dopamine playing an excitatory role [68]. (2) The effects of herbal therapies to regulate the level of the nitric oxide support their contribution towards treating erectile dysfunctions. Nitric oxide (NO) is an established endogenous mediator of penile erection [64] and its synthesis via NO synthase is mainly concentrated in structures of the brain that are involved in sexual behavior (olfactory bulb, amygdala, septal structures, supraoptic and paraventricular nuclei, etc.) $[69,70]$. NO is a known vasodilator which can increase blood flow to the penis inducing penile erection and to other male reproductive organs facilitating better hormonal accessibility in the same to promote robust reproductive functioning [71]. (3) Herbs can favourably interfere in the HPG axis in the regulation of sex hormones importantly testosterone, LH, FSH and interstitial hormones as well as their cross- talks which synchronise male reproductive functioning, such as, the development of secondary male sexual organs, accentuate pubertal changes and increase overall male fertility [72].

\section{Conclusion}

Vajikarana or aphrodisiac herbs can be used as stimulants or tonics to improve male reproductive vitality. Stimulants to reproductive functions are typically heating herbs having an invigorating action on the male sexual organs. Herbal tonics provide better nourishment to the reproductive tissues to rejuvenate the quality and quantity of the same. Some herbs have both stimulating as well as nourishing properties. Owing to the health complications, partial impact and high cost of modern therapies for male infertility, the herbal aphrodisiacs are regaining popularity and laying their long-term impact on male reproductive health by improving sexual behavior, vigor, neuroendocrine control as well as structural and functional aspects of reproductive tissues, qualitative and quantitative improvement in semen and also sperm morphology and motility. Biomedical research should pierce deeper to emerge with more hidden mechanisms by which innumerable herbs may act to eradicate male infertility which is becoming a major threat to upcoming progenies.

\section{References}

1. Sengupta P (2014) Current trends of male reproductive health disorders and the changing semen quality. Int J Prev Med 5: 1-5. [Crossref]

2. Sengupta P (2014) Recent trends in male reproductive health problems. Asian J Pharm Clin Res 7:1-5.

3. Sengupta $P$ (2015) Reviewing reports of semen volume and male aging of last 33 years: From 1980 through 2013. Asian Pac J Reprod 4:242-6.

4. Sengupta P, Borges E Jr, Dutta S, Krajewska-Kulak E (2018) Decline in sperm count in European men during the past 50 years. Hum Exp Toxicol 37: 247-255. [Crossref]

5. Sengupta P, Nwagha U, Dutta S, Krajewska-Kulak E, Izuka E (2017) Evidence for decreasing sperm count in African population from 1965 to 2015. Afr Health Sci 17:418-27.

6. Sengupta P, Dutta S, Tusimin MB, Irez T, Krajewska-Kulak E, (2018) Sperm counts in Asian men: Reviewing the trend of past 50 years. Asian Pac J Reprod.

7. Sengupta P, Dutta S, Krajewska-Kulak E (2017) The disappearing sperms: analysing the reports published between 1980 and 2015. Am J Men's Health 11:1279-1304.

8. Malviya N, Jain S, Gupta VB, Vyas S (2011) Recent studies on aphrodisiac herbs for the management of male sexual dysfunction--a review. Acta Pol Pharm 68: 3-8 [Crossref] 
9. Low W, Tan H (2007) Asian traditional medicine for erectile dysfunction. The J Men's Health Gend 4:245-250.

10. Agarwal A, Sekhon LH (2011) Oxidative stress and antioxidants for idiopathic oligoasthenoteratospermia: Is it justified? Indian J Urol 27: 74-85. [Crossref]

11. Meeker JD, Godfrey-Bailey L, Hauser R (2007) Relationships between serum hormone levels and semen quality among men from an infertility clinic. $J$ Androl 28: 397-406. [Crossref]

12. Schrag SD, Dixon RL (1985) Occupational exposures associated with male reproductive dysfunction. Annu Rev Pharmacol Toxicol 25: 567-592.

13. Mahfouz R, Sharma R, Sharma D, Sabanegh E, Agarwal A (2009) Diagnostic value of the total antioxidant capacity (TAC) in human seminal plasma. Fertil Steril 91: 805-811.

14. Saleh RA, Agarwal A (2002) Oxidative stress and male infertility: from research bench to clinical practice. $J$ Androl 23: 737-752.

15. Dandona P, Rosenberg MT (2010) A practical guide to male hypogonadism in the primary care setting. Int J Clin Pract 64: 682-696. [Crossref]

16. Coviello AD, Bremner WJ, Matsumoto AM, Herbst KL, Amory JK, et al. (2004) Intratesticular testosterone concentrations comparable with serum levels are not sufficient to maintain normal sperm production in men receiving a hormonal contraceptive regimen. $J$ Androl 25: 931-938.

17. Amory JK, Wang C, Swerdloff RS, Anawalt BD, Matsumoto AM, et al. (2007) The effect of 5alpha-reductase inhibition with dutasteride and finasteride on semen parameters and serum hormones in healthy men. J Clin Endocrinol Metab 92: 1659-1665.

18. Büchter D, Behre HM, Kliesch S, Nieschlag E (1998) Pulsatile GnRH or human chorionic gonadotropin/human menopausal gonadotropin as effective treatment for men with hypogonadotropic hypogonadism: a review of 42 cases. Eur $J$ Endocrinol 139: 298-303. [Crossref]

19. Dass V (2007) Ayurvedic herbs for male reproductive problems. http://www. bluelotusayurveda.com/oldsite/male-herbs-art.html. [Last accessed on September 17, 2017].

20. Chauhan NS, Sharma V, Dixit VK, Thakur M (2014) A review on plants used for improvement of sexual performance and virility. Biomed Res Int 2014: 868062. [Crossref]

21. Misra DS, Maiti RK, Bera S, Das K, Ghosh D (2005) Protective effect of composite extract of Withania somnifera, Ocimum sanctum and Zingiber officinale on swimminginduced reproductive Endocrine dysfunctions in male rat. Iranian J Pharmacol Therap 4: $110-117$.

22. Iuvone T, Esposito G, Capasso F, Izzo AA (2003) Induction of nitric oxide synthase expression by Withania somnifera in macrophages. Life Sci 72: 1617-1625. [Crossref]

23. Ambiye VR, Langade D, Dongre S, Aptikar P, Kulkarni M, et al. (2013) Clinica Evaluation of the Spermatogenic Activity of the Root Extract of Ashwagandha (Withania somnifera) in Oligospermic Males: A Pilot Study. Evid Based Complement Alternat Med 2013: 571420. [Crossref]

24. Ahmad MK, Mahdi AA, Shukla KK, Islam N, Rajender S, et al. (2010) Withania somnifera improves semen quality by regulating reproductive hormone levels and oxidative stress in seminal plasma of infertile males. Fertil Steril 94: 989-996.

25. Mahdi AA, Shukla KK, Ahmad MK, Rajender S, Shankhwar SN, et al. (2009) Withania somnifera improves semen quality in stress-related male fertility. Evid-Based Complement Alternat Med 2011: 576962.

26. Sengupta P, Agarwal A, Pogrebetskaya M, Roychoudhury S, Durairajanayagam D, et al. (2018) Role of Withania somnifera (Ashwagandha) in the management of male infertility. Reprod Biomed Online 36: 311-326. [Crossref]

27. Longhi JG, Perez E, deLima JJ, Candido LMB (2011) In vitro evaluation of Mucuna pruriens (L.) DC. antioxidant activity. Braz J Pharm Sci 47:535-544.

28. Saksena S, Dixit VK (1987) Role of total alkaloids of Mucuna pruriens Baker in spermatogenesis in Albino rats. Indian J Nat Prod 3:3-7.

29. Amin YMN, Rehman ZS, Khan NA (1996) Sexual function improving effect of M. pruriens in sexually normal male rats. Fitoterapial 67:53-58.

30. Suresh S, Prithiviraj E, Prakash S (2010) Effect of Mucuna pruriens on oxidative stress mediated damage in aged rat sperm. Int $J$ Androl 33: 22-32. [Crossref]

31. Singh AP, Sarkar S, Tripathi M, Rajender S (2013) Mucuna pruriens and its major constituent L-DOPA recover spermatogenic loss by combating ROS, loss of mitochondrial membrane potential and apoptosis. PLoS One 8: e54655. [Crossref]
32. Suresh S, Prakash S (2012) Effect of Mucuna pruriens (Linn.) on sexual behavior and sperm parameters in streptozotocin-induced diabetic male rat. J Sexual Med 9:3066-3078.

33. Ahmad MK, Mahdi AA, Shukla KK, Islam N, Jaiswar SP, et al. (2008) Effect of Mucuna pruriens on semen profile and biochemical parameters in seminal plasma of infertile men. Fertil Steril 90:627-635.

34. Shukla KK, Mahdi AA, Ahmad MK, Jaiswar SP, Shankwar SN, et al. (2010) Mucuna pruriens reduces stress and improves the quality of semen in infertile men. Evid-Based Complement Alternat Med 7:137-144.

35. Shukla KK, Mahdi AA, Shankwar SN, Ahmad MK (2008) Effect of Mucun apruriens on hormonal status and semen quality in infertile males. Contraception 78:194.

36. Tocharus C, Jeenapongsa R, Teakthong T, Smitasiri Y (2005) Effects of long-term treatment of Butea superba on sperm motility and concentration. Naresuan Univ $J$ 13:11-17.

37. Manosroi A1, Sanphet K, Saowakon S, Aritajat S, Manosroi J (2006) Effects of Butea superba on reproductive systems of rats. Fitoterapia 77: 435-438. [Crossref]

38. Cherdshewasart W, Nimsakul N (2003) Clinical trial of Buten superba, an alternative herbal treatment for erectile dysfunction. Asian J Androl 5:243-246.

39. Chauhan NS, Rao ChV, Dixit VK (2007) Effect of Curculigo orchioides rhizomes on sexual behaviour of male rats. Fitoterapia 78: 530-534. [Crossref]

40. Chauhan NS, Dixit VK (2008) Spermatogenic activity of rhizomes of Curculigoorchioidesgaertn in male rats. Int J Appl Res Nat Prod 1:26-31.

41. Thakur M, Dixit VK (2007) Effect of some vajikaran herbs on pendiculation activities and in vitro sperm count in male. Sexual Disab 25:203-207.

42. Thakur M, Loeppert R, Praznik M, Dixit VK (2008) Effect of some ayurvedic vajikarana rasayana herbs on heat induced testicular damage in male rats. $J$ Complement Integ Med $5: 1-14$.

43. Abd El-Rahman HA, El-Badry AA, Mahmoud OM, Harraz FA (1999) The effect of the aqueous extract of Cynomorium coccineum on the epididymal sperm pattern of the rat. Phytother Res 13:248-250.

44. Thakur M, Dixit VK (2008) A review on some important medicinal plants of Chlorophytumspp. Pharmacog Rev 2:168-172.

45. Kenjale R, Shah R, Sathaye S (2008) Effects of Chlorophytum borivilianum on sexual behaviour and sperm count in male rats. Phytother Res 22: 796-801. [Crossref]

46. Thakur M, Bhargava S, Praznik W, Loeppert R, Dixit VK (2009) Effect of Chlorophytum borivilianum Santapau and Fernandes on sexual dysfunction in hyperglycemic male rats. Chinese J Integ Med 15:448-453.

47. Vyawahare NS, Kagathara VG, Kshirsagar AD, Rajendran R, Patil MN, et al. (2009) Effect of hydroalcoholic extract of Chlorophytum borivilianum tubers in alleviating the diabetic impotency in streptozotocin induced male diabetic rats. Pharm Res 1:314-319.

48. The state pharmacopoeia commission of PR China, in Pharmacopoeia of The People's Republic of China English Edition, vol.1, Chemical Industry Press, Beijing, China, 2000.

49. Makarova MN, Pozharitskaya ON, Shikov AN, Tesakova SV, Makarov VG, et al. (2007) Effect of lipid based suspension of Epimedium koreanum Nakai extract on sexual behavior in rats. J Ethnopharmacol 114:412-416.

50. Liu W, Xin Z, Xin H, Yuan Y, Tian L, et al. (2005) Effects of icariin on erectile function and expression of nitric oxide synthase isoforms in castrated rats. Asian $J$ Androl 7:381-388.

51. Zhang ZB, Yang QT (2006) The testosterone mimetic properties of icariin. Asian $J$ Androl 8: 601-605. [Crossref]

52. Cyranoski D (2005) Malaysian researchers bet big on home-grown Viagra. Nat Med 11: 912. [Crossref]

53. Ang HH, Sim MK (1998) Eurycoma longifolia increases sexual motivation in sexually naive male rats. Arch Pharm Res 21:779-781.

54. Ang HH1, Sim MK (1997) Eurycoma longifolia Jack enhances libido in sexually experienced male rats. Exp Anim 46: 287-290. [Crossref]

55. Chopra RN, Chopra IC, Handa KL, Kapur LD (1958) Chopra's Indigenous Drugs of India. UN Dhur and Sons Private Limited, Calcutta, India, 2nd edition, 1958.

56. Georgiev P, Dimitrov M, Vitanov S (1988) Effect of Tribestan (from Tribulus terrestris) on plasma testosterone and spermatogenesis in male lambs and rams. Veter Sbirka 86:20-22. 
57. Koumanov F, Bozadjieva E, Andreeva M (1982) Clinical trial of the drug Tribestan. Savremenna Med 33:211-215.

58. Gauthaman K, Adaikan PG, Prasad RNV, Goh VHH, Ng SC (2000) Changes in hormonal parameters secondary to intravenous administration of Tribulus terrestris extract in primates. Int $J$ Impotence Res 1:6.

59. Adaikan PG, Gauthaman K, Prasad RNV, Ng SC (2000) Proerectile pharmacological effects of Tribulus terrestris extract on the rabbit corpus cavernosum. Ann Acad Med Singapore 29:22-26.

60. Gauthaman K, Adaikan PG, Prasad RN (2002) Aphrodisiac properties of Tribulus Terrestris extract (Protodioscin) in normal and castrated rats. Life Sci 71: 1385-1396. [Crossref]

61. Gauthaman K, Adaikan PG (2005) Effect of Tribulus terrestris on nicotinamide adenine dinucleotide phosphate-diaphorase activity and androgen receptors in rat brain. $J$ Ethnopharmacol 96:127-132.

62. Gauthaman K, Ganesan AP (2008) The hormonal effects of Tribulus terrestris and its role in the management of male erectile dysfunction--an evaluation using primates, rabbit and rat. Phytomedicine 15: 44-54. [Crossref]

63. Gauthaman K, Ganesan AP, Prasad RNV (2003) Sexual effects of puncturevine (Tribulus terrestris) extract (protodioscin): an evaluation using a rat model. J Alt Comp Med 9:257-265.
64. Achike FI, Kwan CY (2003) Nitric oxide, human diseases and the herbal products that affect the nitric oxide signalling pathway. Clin Exp Pharmacol Physiol 30(9):605-615.

65. Sengupta P, Banerjee R2, Nath S3, Das S2, Banerjee S2 (2015) Metals and female reproductive toxicity. Hum Exp Toxicol 34: 679-697. [Crossref]

66. Bhattarai T, Chaudhuri P, Bhattacharya K, Sengupta P (2014) Effect of progesterone supplementation on post-coital unilaterally ovariectomized superovulated mice in relation to implantation and pregnancy. Asian J Pharm Clin Res 7:29-31.

67. Krajewska-Kulak E, Sengupta P (2013) Thyroid function in male infertility. Front Endocrinol (Lausanne) 4: 174. [Crossref]

68. Argiolas A, Melis MR (2013) Neuropeptides and central control of sexual behaviour from the past to the present: a review. Prog Neurobiol 108: 80-107. [Crossref]

69. Anderson KE (1993) Pharmacology of lower urinary tract smooth muscles and penile erectile tissues. Pharmacol Rev 45: 253-308. [Crossref]

70. Chaudhuri P, Bhattacharya K, Sengupta P (2014) Misty role of amygdala in female reproductive behavior. Int J Pharm Pharm Sci 6:563-564.

71. Nehra A, Colreavy F, Khandheria BK, Chandrasekaran K (2001) Sildenafil citrate, selective phosphodiesterase type 5 inhibitor: urologic and cardiovascular implications. World J Urol 19: 40-45.

72. Dohle GR, Smit M, Weber RF (2003) Androgens and male fertility. World J Urol 21:341-345.

Copyright: (C2018 Dutta S. This is an open-access article distributed under the terms of the Creative Commons Attribution License, which permits unrestricted use, distribution, and reproduction in any medium, provided the original author and source are credited. 